\title{
REMOÇÃO DE MONÓXIDO DE CARBONO COM SISTEMA ALTERNATIVO
}

\author{
Lucimara Gonçalves Maia, Reinaldo Aparecido Bariccatti, Cleber Antônio Lindino *

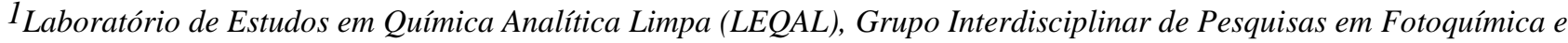 \\ Eletroquímica Ambiental (GIPEFEA), Universidade Estadual do Oeste do Paraná,85903-000, Toledo, Paraná, Brasil.
}

E-mail*: lindino99@gmail.com

O monóxido de carbono $(\mathrm{CO})$ é responsável por diversas intoxicações em ambientes domiciliares e industriais, principalmente em sistemas de combustão mal dimensionados ou com manutenção precária. A transformação da biomassa em gás de síntese (syngas) gera quantidade significativa de CO necessitando, assim, de tratamento adequado. Uma das maneiras de diminuir o risco de intoxicação causado pelo monóxido de carbono é a sua conversão para dióxido de carbono $\left(\mathrm{CO}_{2}\right)$ em processo de oxirredução. Neste trabalho utilizou-se solução de permanganato de potássio $\left(\mathrm{KMnO}_{4}\right)$, estudando-se diferentes temperaturas, concentrações e tempo de reação para a remoção do $\mathrm{CO}$. Os resultados obtidos com $5 \mathrm{~mL}$ de $\mathrm{KMnO}_{4}$ na concentração de $0,02 \mathrm{~mol} \mathrm{~L}^{-1}$ e $10 \mathrm{~mL}$ de $\mathrm{CO}$ mostram que é possível a remoção de $82,9 \%$ na temperatura de $35^{\circ} \mathrm{C}$ em 90 minutos com cinética de pseudoprimeira ordem. A oxidação do monóxido de carbono por permanganato mostrou-se ser uma alternativa viável e de baixo custo, podendo ser utilizada na purificação do ar no interior das indústrias e residências que tem como contaminante o CO.

Palavras-chave: Gás de síntese. Oxidação. Segurança Ocupacional.

\section{Introdução}

De acordo com a Agência Internacional de Energia (IEA), as tecnologias baseadas em biomassa são as mais importantes fontes renováveis e uma das melhores alternativas para a indústria reduzir a dependência de matérias primas fósseis e diminuir as emissões de gases do efeito estufa [1].

Uma imensa quantidade gerada de resíduos de biomassa proveniente de diferentes fontes rurais e urbanas traz a necessidade de processos de reaproveitamento que possam minimizar o impacto ambiental destes materiais e, ao mesmo tempo, gerar energia ou matérias primas para outros processos. Além disso, a utilização de biomassa é um processo sustentável e que desempenha importante papel ambiental e econômico [2].

Há várias rotas possíveis para conversão da biomassa em outros produtos, que incluem a química, a bioquímica, a termoquímica e as rotas mecânicas. Uma das mais promissoras tecnologias para converter a biomassa é a gaseificação, um processo termoquímico que envolve a oxidação parcial de uma fonte de carbono, tal como carvão, gás natural ou biomassa, para produção de gás combustível conhecido como gás de síntese ou syngas [3-4].

O gás de síntese é uma mistura de gases que ocorre por meio da oxidação parcial da biomassa sob temperaturas entre 800 a $900{ }^{\circ} \mathrm{C}$. O gás produzido é considerado de baixo poder calorífico, o qual produz cerca de 4 a $6 \mathrm{MJ}$ para cada $\mathrm{Nm}^{3}$ (normal metro cúbico) de gás gerado [5], queimado diretamente com a finalidade da geração de energia térmica ou usado como um combustível para motores a gás e turbinas. O syngas também é matéria-prima na indústria química [6].

O gás proveniente da gaseificação é composto, principalmente, de monóxido de carbono $(\mathrm{CO})$, hidrogênio $\left(\mathrm{H}_{2}\right)$, metano $\left(\mathrm{CH}_{4}\right)$, com pequenas quantidades de dióxido de carbono $\left(\mathrm{CO}_{2}\right)$, nitrogênio $\left(\mathrm{N}_{2}\right)$, vapor de água $\left(\mathrm{H}_{2} \mathrm{O}\right)$, gás sulfídrico $\left(\mathrm{H}_{2} \mathrm{~S}\right)$, gás amônia $\left(\mathrm{NH}_{3}\right)$, além de outros hidrocarbonetos, cujas proporções variam de acordo com o tipo de biomassa e as condições do processo de gaseificação, particularmente o ar ou o oxigênio que está sendo usado na oxidação [7]. Na gaseificação, a porcentagem média de $\mathrm{CO}$ é de $20-30 \%$ mostrando a importância de metodologias para adsorver ou converter este gás, bem como aumentando o poder calorífico do gás de síntese [8-9].

Outras fontes de emissão do CO são a queima de combustíveis fósseis em ambientes fechados ou mal ventilados, em aquecedores com defeitos, em incêndios e em alguns setores industriais [10-12]. Nesse sentido, estudos que visem sua eliminação são importantes.

O monóxido de carbono é um gás insípido e inodoro, incolor, não irritante, inflamável e tóxico aos seres humanos e animais [13-15]. A intoxicação por monóxido de carbono pode ser fatal, pois é absorvido rapidamente pelo epitélio pulmonar e por possuir interação 200 a 270 vezes maior com a hemoglobina $(\mathrm{Hb})$ do que com o oxigênio $\left(\mathrm{O}_{2}\right)$, para formar o complexo de carboxiemoglobina $(\mathrm{HbCO})$, que impede o transporte de oxigênio pelo corpo [16].

Os principais sintomas de intoxicação são confusão mental, insônia, tremor, perda da audição, dor de cabeça, tonturas, fraqueza, náuseas, convulsões, anóxia, problemas no sistema nervoso, insuficiência renal, colapso cardiovascular [17- 
18] e pode haver o aumento do risco da síndrome da morte súbita infantil, além do transtorno de déficit de atenção [19-21]. Quando os níveis de $\mathrm{HbCO}$ atingem entre $30 \%$ e $70 \%$ resulta na perda de consciência e, eventualmente, leva ao óbito [22]. Aproximadamente $80 \%$ das mortes com gases estão relacionadas à intoxicação com $\mathrm{CO}$ [23].

Também há estudos sobre os efeitos em longo prazo da exposição ao $\mathrm{CO}$, podendo causar diversas sequelas neurológicas retardadas (DNS), por exemplo, a desmineralização difusa no cérebro acompanhada por letargia, alterações de comportamento, depressão, ansiedade, perda de memória, características do mal de Parkinson e doença isquêmica, sendo que apenas $60 \%$ dos pacientes com DNS conseguem se recuperar dentro de 1 ano [19, 22, 24] Por isso, a contaminação desse gás constitui um problema social relevante.

Uma maneira de diminuir o impacto ambiental causado pelo monóxido de carbono é a conversão deste em dióxido de carbono $\left(\mathrm{CO}_{2}\right)$ que, apesar de poder contribuir em longo prazo para o aumento do efeito estufa, constitui-se num gás muito menos agressivo do que o monóxido de carbono que o origina [25].

Os materiais mais utilizados na remoção ou conversão de monóxido de carbono são os carvões ativados, as zeólitas, as estruturas porosas de metais orgânicos e os catalisadores metálicos (Pt, Pd e Au etc.) [26]. Os catalisadores metálicos são materiais que têm sido amplamente utilizados para a oxidação do CO por várias décadas, devido ao seu elevado desempenho catalítico. No entanto, o alto custo e a baixa estabilidade térmica têm levado as pesquisas para outros catalisadores com menor custo e mais abundantes [25, 27].

Os catalisadores de óxidos de metais de transição apresentam um baixo custo e uma elevada estabilidade térmica, sendo considerados candidatos potenciais para remoção do monóxido de carbono [27-28].

Particularmente, o dióxido de manganês $\left(\mathrm{MnO}_{2}\right)$ tem sido amplamente estudado em catalisadores, supercapacitores, degradação de corantes e materiais para baterias, pois possui potencial para atuar no processo de oxidação devido à sua capacidade de armazenar elétrons. Isso pelo fato desse dióxido possuir ciclos de oxidação e redução bem definidos, bem como por sua interação como agentes redutores e oxidantes. Além disso, é um composto que possui compatibilidade com o meio ambiente, abundância natural e baixo custo [25-31].

Contudo, na preparação do catalisador $\mathrm{MnO}_{2}$ pode-se obter diferentes formas estruturais nanoparticuladas como $\alpha$-, $\beta$-, $\gamma$-, $\delta$ - e $\varepsilon-\mathrm{MnO}_{2}$ [26-30] e o desempenho catalítico depende dessas formas cristalográficas. Por isso, a preparação desse catalisador demanda um controle nas fases cristalinas das nanopartículas, que acaba inibindo a sua utilização em aplicações práticas para a oxidação catalítica [30-31].

Já o permanganato de potássio $\left(\mathrm{KMnO}_{4}\right)$ é um composto que tem sido amplamente utilizado no tratamento de poluentes de água potável e águas residuais há mais de 50 anos e é considerado um dos oxidantes mais importantes em reações orgânicas e inorgânicas [32].

Suas reações são reguladas pelo $\mathrm{pH}$ do meio. Os estados de oxidação do manganês entre $+2 \mathrm{a}+7$ apresentam potenciais de redução padrão em meio ácido de $+1,69 \mathrm{~V}$ para o par $\mathrm{Mn}$ (VII)/Mn (VI) e +1,51 V para o par Mn (VII)/Mn(II) [33]. Em relação ao custo, em média, o preço de $1 \mathrm{~kg}$ de permanganato de potássio é de $\mathrm{R} \$ 53,00$ [34].

Considerando o caráter redutor do monóxido de carbono e o caráter oxidante do permanganato de potássio, assim como o baixo custo deste último, este trabalho propôs remover o $\mathrm{CO}$, visando aplicação em ambientes contaminados, como usinas de gaseificação, indústrias e sistemas com aquecedores a gás.

\section{Parte Experimental}

\subsection{Soluções e reagentes}

O monóxido de carbono foi coletado diretamente do cilindro do gás (White Martins - 99,5\%) por meio de bag de Tedlar $^{\circledR}$ (fluoreto de polivinila), de volume total de 1 litro, com mangueira. O gás foi coletado abrindo-se a dupla válvula do cilindro e injetando-se lentamente no bag até o limite de volume do mesmo.

Todos os reagentes químicos utilizados foram de grau de pureza para análise. A água utilizada para o preparo de soluções padrão ou diluições de amostras foi destilada e purificada por osmose reversa (Adamo, resistência da água de $5 \mathrm{M} \Omega \mathrm{cm}^{-1}$ a 25 $\left.{ }^{\circ} \mathrm{C}\right)$.

A solução utilizada para os estudos de remoção do $\mathrm{CO}$ foi preparada com $\mathrm{KMnO}_{4}$ (Nuclear - 99\%) em água purificada com adição de $27 \mathrm{~mL}$ de $\mathrm{H}_{2} \mathrm{SO}_{4}$ concentrado (Alphadec - 97\%), para manter o pH em 1,0. Para eliminar impurezas, como o dióxido de manganês $\left(\mathrm{MnO}_{2}\right)$, que acelera a decomposição da referida solução, ela ficou em repouso por um período de cinco dias e, em seguida, filtrou-se com lã de vidro, sendo colocada em frascos protegidos da luz.

\subsection{Estudos de remoção}

Os testes foram realizados em seringas hipodérmicas de polipropileno com volume de $60 \mathrm{~mL}$, recobertas por fita isolante preta, para evitar a fotodecomposição das soluções.

Em cada teste inseriu-se na seringa o volume de $5 \mathrm{~mL}$ de solução de permanganato de potássio nas concentrações de $0,0005 \mathrm{~mol} \mathrm{~L}^{-1}$ ou $0,02 \mathrm{~mol} \mathrm{~L}^{-1} \mathrm{e}$ volumes diferentes de monóxido de carbono, a partir do bag, sendo colocadas em um banho termostatizado (Solab, $\pm 1^{\circ} \mathrm{C}$ ) nas temperaturas de teste de 15 a $35^{\circ} \mathrm{C}$, por período de 15,30 ou 90 minutos, com ou sem agitação. Após os períodos de reação mediu-se a absorvância de cada amostra em espectrofotômetro UV-visível (PG instruments $\mathrm{T} 80+$ ) na região entre $400 \mathrm{~nm}$ a $700 \mathrm{~nm}$ com cubetas de quartzo e caminho óptico igual a 1,00 cm. Para as amostras que foram 
agitadas utilizou-se o agitador orbital em $200 \mathrm{rpm}$ (Orbit microprocessor ShakerBath, LabLine). Na Tabela 1 estão dispostos os parâmetros utilizados para as demais análises.

Tabela 1 - Parâmetros utilizados nos testes de remoção de CO.

\begin{tabular}{|c|c|c|c|c|}
\hline $\begin{array}{c}\text { Volume (mL) } \\
\text { da solução de } \\
\mathrm{KMnO}_{4} 5 \times 10^{-4} \\
\mathrm{~mol} \mathrm{~L}^{-1}\end{array}$ & $\begin{array}{c}\text { Volume (mL) } \\
\text { da solução de } \\
\mathrm{KMnO}_{4} 2 \times 10^{-} \\
{ }^{2} \mathrm{~mol} \mathrm{~L}^{-1}\end{array}$ & CO (mL) & $\begin{array}{c}\text { Tempo } \\
\text { (min) }\end{array}$ & $\begin{array}{c}\text { Temperatura } \\
\left({ }^{\circ} \mathrm{C}\right)\end{array}$ \\
\hline $\begin{array}{l}5,10,15,20, \\
25,30,35,40\end{array}$ & - & 5 & 15,30 & 25 \\
\hline 5 & - & $\begin{array}{c}5,10,15 \\
20,25,30 \\
35,40,45 \\
50,55\end{array}$ & 15,30 & 25 \\
\hline- & 5 & $\begin{array}{c}5,10,15 \\
20,25\end{array}$ & $\begin{array}{c}15,30 \\
90\end{array}$ & $\begin{array}{l}15,20,25, \\
30,35\end{array}$ \\
\hline- & 5 & 5 & $1440 *$ & 25 \\
\hline
\end{tabular}

* A amostra ficou em agitação por duas horas e, em seguida, manteve-se por vinte duas horas em repouso.

A pressão atmosférica média em Toledo-PR, a qual foi utilizada para o cálculo da quantidade de substância (mol) do monóxido de carbono, foi obtida do Sistema Meteorológico do Paraná [35] referente ao período dos testes, com valor médio de $0,946 \mathrm{~atm}$ e amplitude de 0,003 atm. Os gráficos foram construídos pelo programa software Origin ${ }^{\circledR} 8.0$, a partir do pico de maior absorção da solução de permanganato de potássio no comprimento de onda de $523 \pm 1 \mathrm{~nm}$. Estes dados foram utilizados nos cálculos de quantidade de monóxido de carbono por meio da Equação de Van der Waals para gases reais.

\subsection{Estudo cinético}

A partir dos resultados obtidos com diferentes temperaturas, obteve-se a melhor condição para os estudos cinéticos, utilizando-se $5 \mathrm{~mL}$ de solução $0,02 \mathrm{~mol} \mathrm{~L}^{-1}$ de permanganato de potássio e $10 \mathrm{~mL}$ de monóxido de carbono em agitação orbital $(200 \mathrm{rpm})$, na temperatura de $35^{\circ} \mathrm{C}$, por período de 15, 30, 45, 60, 75, 90, 105 e 120 min. Após cada período de agitação, mediu-se a absorvância de cada amostra, em espectrofotômetro UV-visível (PG instruments T80+) na região entre $400 \mathrm{~nm}$ a $700 \mathrm{~nm}$ com cubetas de quartzo e caminho óptico igual a $1,00 \mathrm{~cm}$. Todos os experimentos foram realizados em duplicata.

\subsection{Análise de interferência}

Para verificar a possível interferência de outros gases na reação com permanganato de potássio foram estudados os gases hidrogênio (Air Liquide - 99,99\%), nitrogênio (Linde 99,999\%), metano (AGA - 99,995\%), dióxido de carbono (White Martins - 99,99\%) e o monóxido de carbono (White Martins 99,5\%), sendo que o gás hélio (Gama Gases - 99,999\%) foi utilizado para limpar o coletor de gases ( $b a g$ ) entre as medidas.
Nesta análise foram inseridos $10 \mathrm{~mL}\left(3,87 \times 10^{-4} \mathrm{~mol}\right)$ dos gases em $5 \mathrm{~mL}$ de solução de $0,02 \mathrm{~mol} \mathrm{~L}^{-1}$ de permanganato de potássio e submetido à agitação orbital constante $(200 \mathrm{rpm})$, com uma temperatura de $35{ }^{\circ} \mathrm{C}$ por $1 \mathrm{~h} 30 \mathrm{~min}$. Após o tempo de reação de cada amostra, as mesmas foram analisadas no espectrofotômetro UV-visível nas mesmas condições utilizadas nos testes anteriores, assim como as análises foram realizadas em duplicata.

\section{Resultados e discussões}

\subsection{Estudo da remoção de monóxido}

A Figura 1 apresenta os resultados dos testes com a solução de permanganato na ausência e presença de agitação e a reação com monóxido de carbono, todos a $25{ }^{\circ} \mathrm{C}$, comparados com amostra controle (sem reação).

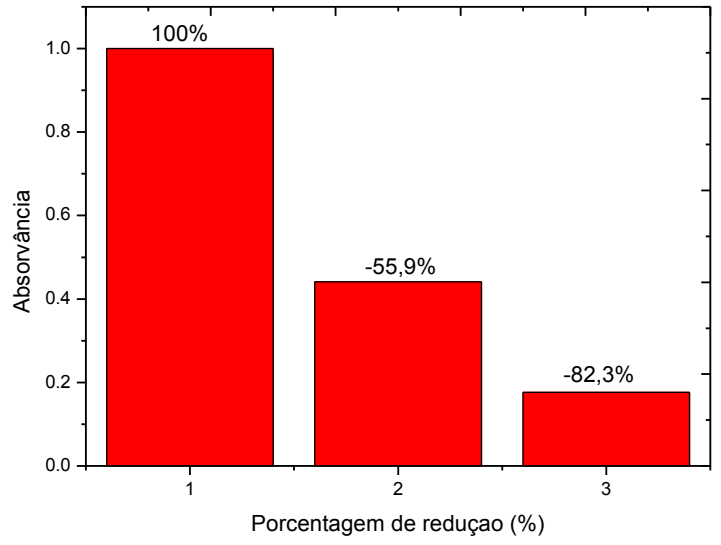

Figura 1 - Resultados da análise realizada com $5 \mathrm{~mL}$ de $0,02 \mathrm{~mol} \mathrm{~L}^{-1} \mathrm{KMnO}_{4}$. Teste 1: branco (controle). Teste 2: $5 \mathrm{~mL}$ de CO sem agitação por período de 24 horas. Teste 3: $5 \mathrm{~mL}$ de $\mathrm{CO}$ com agitação por 2 horas e 22 horas em repouso.

Na Figura 1 observa-se o decréscimo na absorvância da solução de permanganato com a reação com $\mathrm{CO}$ e o efeito da agitação na remoção do monóxido de carbono, fator mantido em todas as análises seguintes. A reação de oxirredução neste processo pode ser expressa pela equação 1

$$
2 \mathrm{MnO}_{4}^{-}(a q)+5 \mathrm{CO}_{(g)}+6 \mathrm{H}_{(a q)}^{+} \leftrightarrows 2 \mathrm{Mn}^{2+}{ }_{(a q)}+5 \mathrm{CO}_{2}(g)+3 \mathrm{H}_{2} \mathrm{O}_{(a q)}
$$

A Figura 2 apresenta os resultados da absorvância normalizada $\left(\mathrm{A} / \mathrm{A}_{0}\right)$ em função da quantidade de substância (mol) do gás $\mathrm{CO}$ e de ar atmosférico, sendo que $\mathrm{A}$ indica o valor observado da absorvância do composto com o gás e $\mathrm{A}_{0}$ é absorvância inicial da solução sem o gás (branco). 


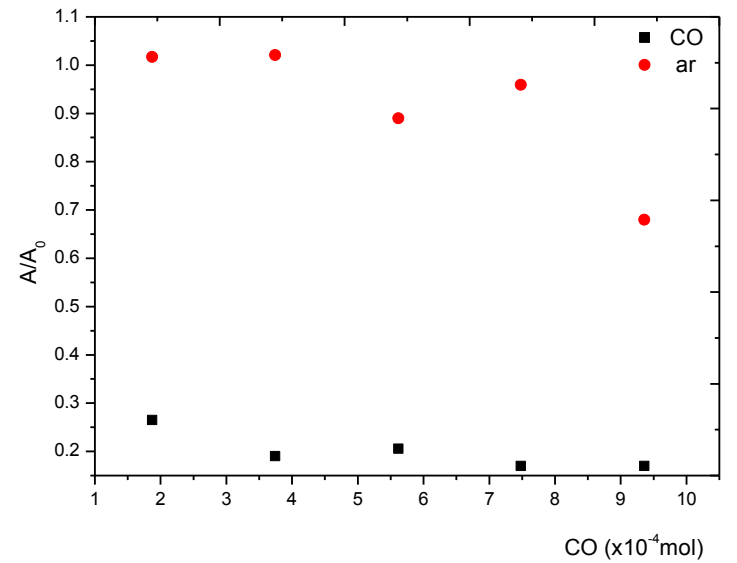

Figura 2 - Testes de remoção com $5 \mathrm{~mL}$ de solução $0,02 \mathrm{~mol} \mathrm{~L}^{-1}$ de $\mathrm{KMnO}_{4}$ com variação na quantidade de $\mathrm{CO}$ comparado com o ar (branco), agitação em banho térmico a $35^{\circ} \mathrm{C}$ por $1 \mathrm{~h} 30 \mathrm{~min}$.

De acordo com os resultados observados na Figura 2 verifica-se que a absorvância inicial da solução de permanganato decai com o aumento na quantidade de monóxido de carbono indicando o processo de reação para formação de $\mathrm{Mn}^{2+}$, que é incolor. A solução com volumes crescentes de ar apresentou variação na absorvância diferente da observada com a inserção de $\mathrm{CO}$, sem interferir no método.

A Tabela 2 apresenta os resultados dos testes de redução na absorvância das soluções de permanganato de potássio com a variação da temperatura, do tempo e da agitação. A Figura 3 apresenta os resultados da variação da temperatura para a reação estudada, com agitação.

Tabela 2 - Resultados da diminuição da absorvância (remoção de CO) obtidos com $5 \mathrm{~mL}$ de solução $0,02 \mathrm{~mol} \mathrm{~L}^{-1} \mathrm{KMnO}_{4}$ e $10 \mathrm{~mL}$ de $\mathrm{CO}$ com e sem agitação em tempo e temperaturas distintos e suas respectivas porcentagens de redução.

\begin{tabular}{|c|c|c|c|c|c|}
\hline \multicolumn{6}{|c|}{ Redução na absorvância } \\
\hline $\begin{array}{c}\text { Temperatura } \\
\left({ }^{\circ} \mathbf{C}\right)\end{array}$ & 15 min* & 15 min** & 30 min $^{*}$ & 30 min** & 1h30min* \\
\hline 20 & $-16,80 \%$ & $-10,70 \%$ & $-16,80 \%$ & $-3,37 \%$ & $-41,10 \%$ \\
\hline 25 & $-13,00 \%$ & $-5,60 \%$ & $-17,80 \%$ & $-15,04 \%$ & $-60,70 \%$ \\
\hline 30 & $-17,00 \%$ & & $-30,50 \%$ & $-15,15 \%$ & $-70,20 \%$ \\
\hline 35 & $-23,90 \%$ & $-0,30 \%$ & $-47,08 \%$ & $-15,74 \%$ & $-82,90 \%$ \\
\hline
\end{tabular}

Estabeleceu-se como condição para os demais estudos da reação a temperatura de $35{ }^{\circ} \mathrm{C}$ com agitação pelo tempo de 1h30min, com diminuição na absorção de $82,9 \%$. Dados não apresentados neste trabalho mostraram que a conversão do $\mathrm{CO}$ não aumenta significativamente $(89 \%)$ em maiores temperaturas e não atendem ao escopo da proposta em remover o $\mathrm{CO}$ em condições menos drásticas.

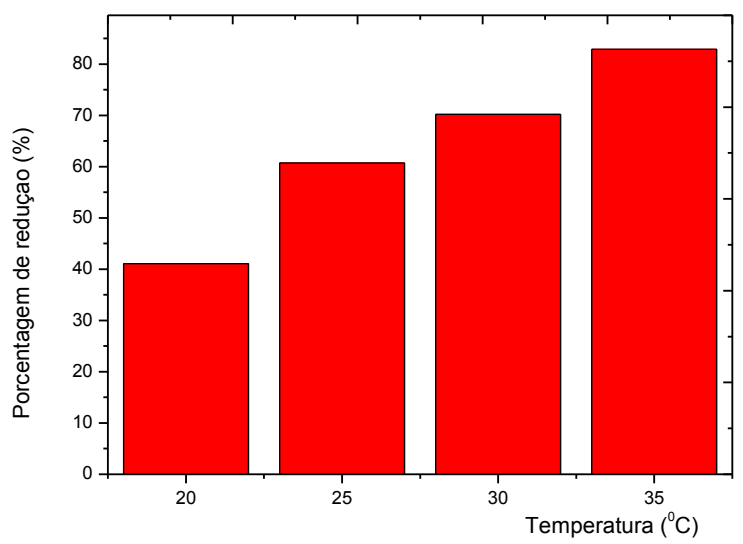

Figura 3 - Porcentagem de redução da absorvância da solução de permanganato de potássio $0,02 \mathrm{~mol} \mathrm{~L}^{-1}$ em função da temperatura. Tempo de agitação de 1 h30min.

A Figura 4 confirma que a melhor condição para a reação entre $\mathrm{o}$ monóxido de carbono e a solução de permanganato ocorre na temperatura de $35^{\circ} \mathrm{C}$, sendo que a partir de $10 \mathrm{~mL}\left(3,87 \times 10^{-4} \mathrm{~mol}\right)$ de monóxido de carbono adicionado, o valor de absorção manteve-se constante, indicando que a reação alcançou o equilíbrio químico na reação.

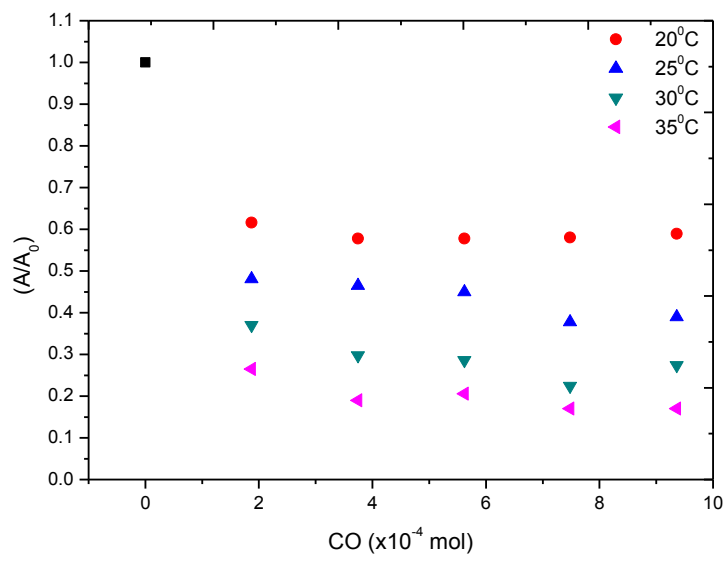

Figura 4 - Absorvância normalizada em função da quantidade de substância de monóxido de carbono com $5 \mathrm{~mL}$ solução de $\mathrm{KMnO}_{4} 0,02 \mathrm{~mol} \mathrm{~L}^{-1}$ e variação da temperatura. Tempo de agitação de $1 \mathrm{~h} 30 \mathrm{~min}$.

Utilizando-se o método da razão molar [36], verificou-se que o ponto de inflexão entre as retas formadas pelo decaimento da absorvância e o patamar do equilíbrio químico indicou a razão estequiométrica da reação com valor médio de 2,12 nas temperaturas estudadas, valor este próximo à razão 
estequiométrica da equação da reação proposta para o permanganato e monóxido de carbono de 2,5 (Equação 1).

Uma vez que o aumento na temperatura provoca $o$ aumento na quantidade de $\mathrm{CO}$ que reage com o $\mathrm{KMnO}_{4}$, deduzse que as reações são endotérmicas, ou seja, a reação é favorecida com o aumento da temperatura.

\subsection{Análise cinética de dados}

A Tabela 3 e a Figura 5 apresentam o decaimento na absorvância nas medidas de $5 \mathrm{~mL}$ de solução de permanganato $0,02 \mathrm{~mol} \mathrm{~L}^{-1}$ com $10 \mathrm{~mL}\left(3,87 \times 10^{-4} \mathrm{~mol}\right)$ de $\mathrm{CO}$, sob agitação e na temperatura de $35^{\circ} \mathrm{C}$, com o tempo.

Tabela 3 - Dados experimentais do teste do estudo da velocidade da reação entre o $\mathrm{CO}$ e a solução de $\mathrm{KMnO}_{4} 0,02 \mathrm{~mol} \mathrm{~L}^{-1}$.

\begin{tabular}{cc}
\hline Absorvância & Tempo (minutos) \\
\hline 0,704 & 0 \\
0,562 & 15 \\
0,448 & 30 \\
0,346 & 45 \\
0,298 & 60 \\
0,275 & 75 \\
0,154 & 90 \\
0,136 & 105 \\
0,092 & 120 \\
\hline
\end{tabular}

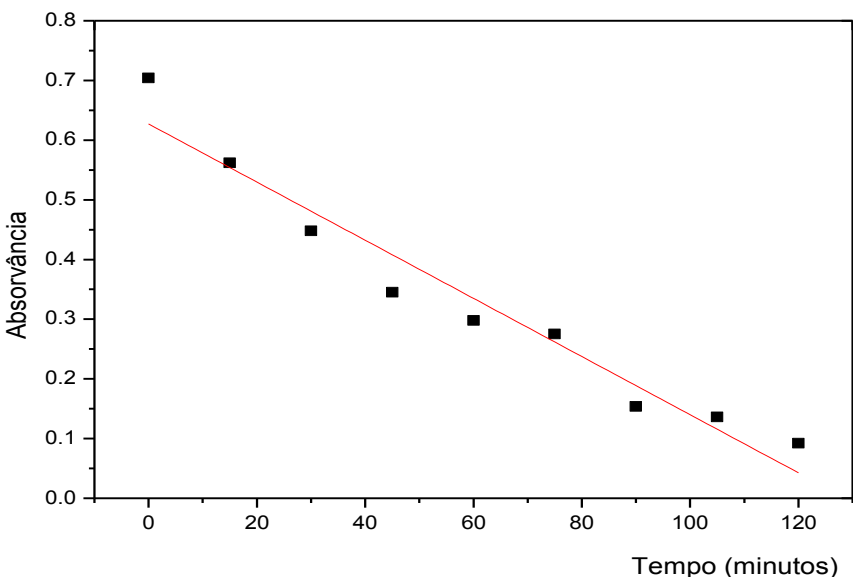

Figura 5 - Curva de decaimento da absorvância da solução de $\mathrm{KMnO}_{4}$ com o tempo a partir da reação com monóxido de carbono, com agitação e temperatura de $35^{\circ} \mathrm{C}$. Equação da reta de $\mathrm{y}=0,62716-0,00487 \mathrm{x}$, com $\mathrm{R}^{2}=0,9440$.

O percentual de remoção de monóxido de carbono em solução de permanganato de potássio aumenta com o tempo de contato. A Figura 6 indica decaimento exponencial em função do tempo, sendo que em 120 minutos removeu-se $86,93 \%$ do $\mathrm{CO}$ presente.

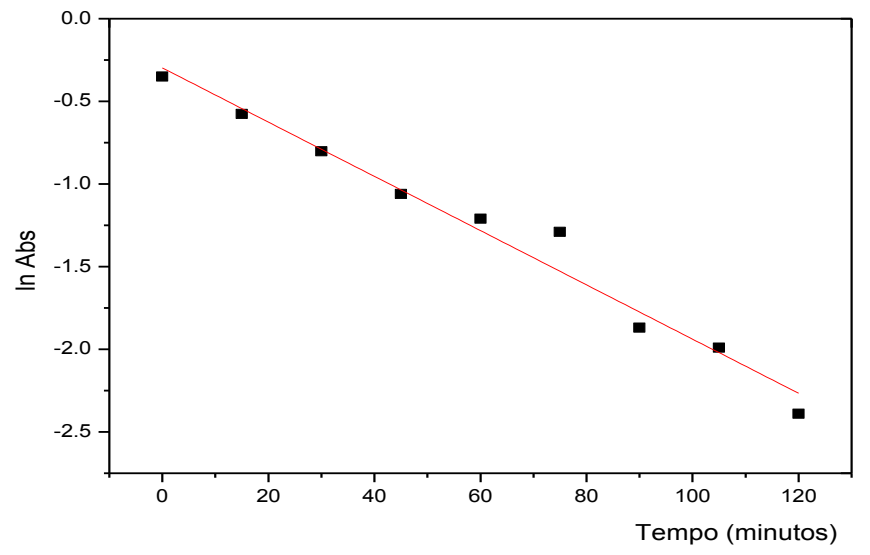

Figura 6 - Curva da função do logaritmo neperiano (ln) para o decaimento da absorvância da solução de $\mathrm{KMnO}_{4}$ com o tempo a partir da reação com monóxido de carbono, com agitação e temperatura de $35{ }^{\circ} \mathrm{C}$. Equação da reta de y = $0,29747-0,01641 x$, com $R^{2}=0,9719$.

De acordo com [36], para a cinética de pseudoprimeira ordem, o logaritmo natural deve diminuir linearmente com o tempo, que pode ser verificado na Figura 6. Na Tabela 4 são apresentados os parâmetros cinéticos para o modelo cinético de pseudoprimeira ordem.

Tabela 4 - Parâmetros do modelo cinético de pseudoprimeira ordem, coeficiente de determinação $\left(R^{2}\right)$, constante de reação $(k)$ e coeficiente linear e seus respectivos modelos

\begin{tabular}{cccc}
\hline Modelo & $\mathbf{R}^{2}$ & $\mathbf{k}\left(\mathbf{m i n}^{-1}\right)$ & Coeficiente linear \\
\hline Abs versus tempo & 0,9440 & $-0,00487$ & $-0,62716$ \\
$\ln$ abs versus tempo & 0,9719 & $-0,01641$ & $-0,29747$ \\
\hline
\end{tabular}

Pode-se observar que o modelo que melhor ajusta a cinética de pseudoprimeira ordem foi o ln abs em função do tempo, com constante de reação $(\mathrm{k})$ obtido pela inclinação da reta com valor de $0,01641 \mathrm{~min}^{-1}$

$\mathrm{O}$ tempo de meia vida $\left(\mathrm{t}_{1 / 2}\right)$ é definido como sendo o tempo necessário, para que se reduza a metade da quantidade inicial e pode ser calculado a partir da equação 2 .

$$
t_{1 / 2}=\frac{\ln 2}{k}=\frac{0,6931}{k}
$$

Observa-se que $t_{1 / 2}$ é independente da concentração inicial e está relacionado somente com a constante de velocidade de pseudoprimeira ordem de reação. Substituindo o valor da constante de reação, que foi obtida experimentalmente, na equação 2 , tem-se que o tempo de meia vida para reação de remoção de $86,93 \%$ de monóxido de carbono, utilizando permanganato de potássio como agente oxidante, é de 42 minutos nas condições estudadas. 


\subsection{Análise de interferência}

Na Tabela 5 e nas Figuras 7 e 8 são apresentados os resultados obtidos da reação da solução de permanganato de potássio $0,02 \mathrm{~mol} \mathrm{~L}^{-1}$ no tempo de $1 \mathrm{~h} 30 \mathrm{~min}$ na temperatura de $35^{\circ} \mathrm{C}$ para os diferentes gases. Os valores do máximo de absorvância foram descontados dos valores de linha base (baseline).

Tabela 5 - Absorbâncias (A) com os diferentes gases e porcentagem da diferença em relação à absorção inicial $\left(\mathrm{A}_{0}\right)$.

\begin{tabular}{ccccccc}
\hline Inicial & Controle & $\mathbf{C O}_{\mathbf{2}}$ & $\mathbf{C H}_{\mathbf{4}}$ & $\mathbf{N}_{\mathbf{2}}$ & $\mathbf{H}_{\mathbf{2}}$ & $\mathbf{C O}$ \\
\hline 0,954 & $0,827^{*}$ & 0,807 & 0,818 & 0,788 & 0,455 & 0,170 \\
Diferença $(\%)$ & 13,31 & 15,41 & 14,25 & 17,40 & 52,31 & 82,18 \\
\hline
\end{tabular}

*solução de permanganato de potássio em agitação por 1h30min.

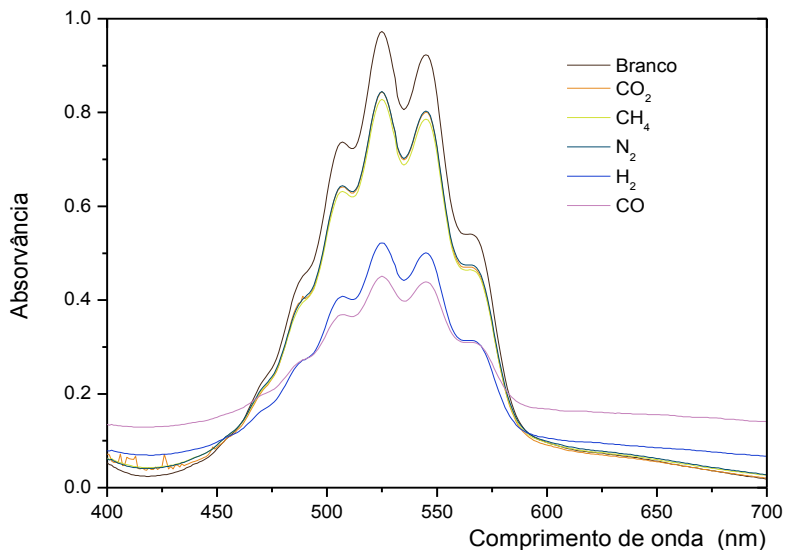

Figura 7 - Espectro da solução de permanganato de potássio com diferentes gases. $\mathrm{O}$ branco (inicial) refere-se à solução de permanganato de potássio sem agitação.

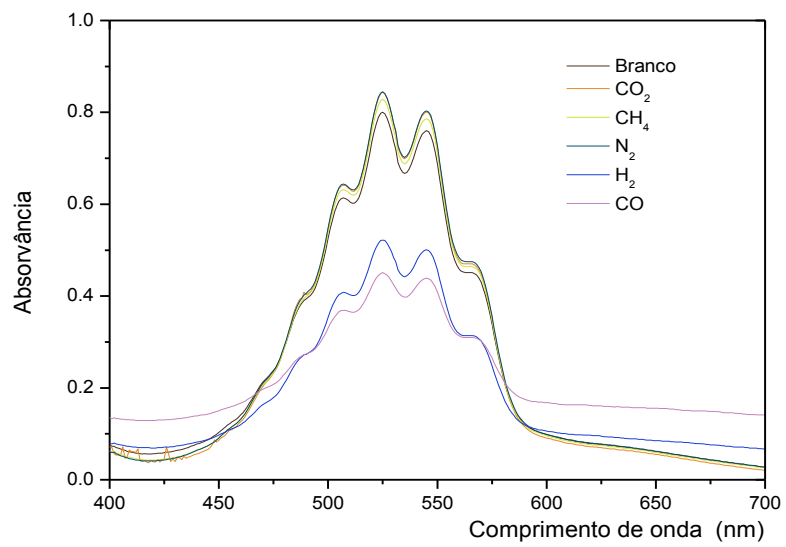

Figura 8 - Espectro eletrônico da solução de permanganato de potássio com diferentes gases. O branco (controle) refere-se à solução de permanganato de potássio em agitação por $1 \mathrm{~h} 30 \mathrm{~min}$.

A diminuição na absorvância entre a solução inicial e o controle foi de $13,31 \%$, sendo que o esperado era próximo de $3 \%$, de acordo com resultados obtidos em outras análises. No entanto, essa diferença está relacionada com a presença de impurezas na solução do permanganato de potássio, particularmente o dióxido de manganês $\left(\mathrm{MnO}_{2}\right)$ que acelera a oxidação da solução.

De acordo com [37-38], é indispensável a eliminação desse contaminante, sendo necessário o repouso da solução por 7 a 15 dias e, posteriormente, a filtração do mesmo por lã de vidro, pois assim elimina possíveis impurezas. Entretanto, nesta análise de interferência de gases, a solução ficou em repouso apenas por quatro dias, podendo ainda ter traços de dióxido de manganês na solução, que atua como catalisador nas reações.

Nota-se pelas Figuras 7 e 8 que os gases de dióxido de carbono, metano e nitrogênio não apresentam diminuição significativa na absorvância da solução, pois esses gases não reagem com solução de permanganato de potássio. Comparandose com o controle, a diferença obtida é de $2,1 \%, 0,84 \%$ e $4,09 \%$, respectivamente.

O gás hidrogênio apresentou diminuição na absorção de $52,31 \%$ indicando que pode interferir na remoção de monóxido de carbono, pois, sendo agente redutor, é oxidado pela solução de permanganato de potássio, como mostra a Equação 3.

$2 \mathrm{MnO}_{4(a q)}^{-}+5 \mathrm{H}_{2(g)}+6 \mathrm{H}_{(a q)}^{+} \leftrightarrow 2 \mathrm{Mn}^{2+}{ }_{(a q)}+8 \mathrm{H}_{2} \mathrm{O}_{(a q)}$

Verificou-se também que a pressão atmosférica local não tem influência significativa na remoção, seja pela pequena variação da mesma ao longo do tempo dos testes, seja pelo seu valor que exerce pouco efeito na Lei de Henry. A solubilidade do CO em água é de $30 \mathrm{mg} \mathrm{L}^{-1}$ a $20^{\circ} \mathrm{C}$ [40].

$\mathrm{Na}$ literatura, a maioria dos trabalhos que investiga a remoção/oxidação/transformação do monóxido de carbono utiliza catalisadores sólidos em alta temperatura [41-43], com eficiência entre 16 e $98 \%$, o que demonstra a aplicabilidade do método proposto neste artigo que utiliza temperaturas mais baixas que as indicadas na literatura.

Em relação ao custo do sistema, um litro de solução de $\mathrm{KMnO}_{4}$ na concentração de $0,02 \mathrm{~mol} \mathrm{~L}^{-1}$ custa $\mathrm{R} \$ 0,17$ e poderia remover $0,05 \mathrm{~mol}$ de $\mathrm{CO}$ ou 1,40 $\mathrm{g}$ ou 1,22 $\mathrm{L}$ nas condições normais de temperatura e pressão (CNTP).

\section{Conclusões}

Os resultados obtidos mostram que a melhor condição para a remoção de $\mathrm{CO}$ com permanganato de potássio na concentração de $0,02 \mathrm{~mol} \mathrm{~L}^{-1}$ ocorre na temperatura de $35^{\circ} \mathrm{C}$, com agitação. Em 1 h30 de reação, consegue-se remover 82,09\% de monóxido de carbono e o gás hidrogênio pode competir nesta reação, uma vez que também é oxidado pelo permanganato. A oxidação do monóxido de carbono por permanganato é uma alternativa viável e de baixo custo, podendo ser utilizada na 
purificação do ar no interior das indústrias e residências que tem como subproduto o CO.

A possibilidade de remoção de $\mathrm{CO}$, de forma simples e barata, diminui ou evita níveis tóxicos deste gás, garantindo a segurança ocupacional em ambientes industriais ou domiciliares.

\section{Agradecimentos}

À UNIOESTE e ao GIPeFEA pelo apoio no desenvolvimento deste trabalho.

\section{REMOVAL OF CARBON MONOXIDE WITH AN ALTERNATIVE SYSTEM}

\begin{abstract}
Carbon monoxide (CO) is responsible for various intoxications in home and industrial environments, especially in poorly-sized or poorly-regulated combustion systems. Another source is the transformation of biomass into synthesis gas, which generates a significant amount of $\mathrm{CO}$ that may require adequate treatment. One way to reduce the risk of poisoning caused by carbon monoxide is its conversion into carbon dioxide $\left(\mathrm{CO}_{2}\right)$ in the process of oxidation. In this work, a solution of potassium permanganate $\left(\mathrm{KMnO}_{4}\right)$ was used at different temperatures and reaction time for the removal of $\mathrm{CO}$. The results obtained with 5 $\mathrm{mL}$ of $0.02 \mathrm{~mol} \mathrm{~L}^{-1} \mathrm{KMnO}_{4}$ and $10 \mathrm{~mL}$ of $\mathrm{CO}$ show that it is possible to remove $82.9 \%$ at $35^{\circ} \mathrm{C}$ in 90 minutes with pseudofirst order kinetics. Oxidation of carbon monoxide by permanganate has proved to be a viable and low-cost alternative and can be used to purify the air inside the industries and residences contaminated with $\mathrm{CO}$.
\end{abstract}

Keywords: Syngas. Oxidation. Occupational Safety.

\section{Referências}

[1] IEA. World Energy Outlook 2013. International Energy Agency (IEA). Paris, France, 2013.2 Disponível em https://www.iea.org/publications/freepublications/publication/WEO2013.pdf. Acesso em 15/06/2016.

[2] IEA. Energy Technology Perspectives 2012. Pathways to a Clean Energy System. ETP 2012. International Energy Agency (IEA), Paris, France, 2012.ISBN: 978-92-64-17488-7.

[3] ARVIDSSON, M.; MORANDIN, M.; HARVEY, S.; Biomass gasificationbased syngas production for a conventional oxo synthesis plant-greenhouse gas emission balances and economic. Journal of Cleaner production, Vol. 99, p. 192205,2015 .

[4] KHONDE, R.; CHAURASIA, A.; Rice husk gasification in a two-stage fixedbed gasifier: production of hydrogen rich syngas and kinetics. Journal of Hydrogen Energy, Vol. 41, n. 21, p. 8793-8802, 2016.
[5] HARYANTO, A.; FERNANDO, S. D.; PORDESIMO, L. O.; ADHIKARI, S.; Upgrading of syngas derived from biomass gasification: A thermodynamic analysis. Biomass and Bioenergy, Vol. 33, n. 5, p. 882-889, 2009

[6] OKAMURA, L. A.; TOMAZZONI, G.; LOPES, E. J.; COSTA, P. R. N.; Obtenção sustentável de gás de síntese: prospecção das tecnologias disponíveis baseada em patentes e artigos. In: III CONGRESSO BRASILEIRO DE PROSPECÇÃO TECNOLÓGICA, 2013, Bahia, Salvador. Anais...Salvador, Vol. 6, n. 1, p. 27-35, 2013. ISSN 1983-1358.

[7] FILIPPIS, P. D.; SCARSELLA, M.; CAPRARIIS, B.; UCCELLARI, R.; Biomass gasification plant and syngas clean-up system. Energy Procedia, Clean, Efficient and Affordable Energy for a Sustainable Future: The 7th International Conference on Applied Energy (ICAE2015), Guiglia (MO), Italian, v. 75, p. 240$245,2015$.

[8] FIGUEIREDO, F. L.; FERNANDES, F.; PETRUCCI, A. L.; FURINI FILHO, R.; MARSURA, M.; Produção de energia elétrica através da biomassa em sistema de gaseificação concorrente e grupo gerador com capacidade de 50 kVA. Semina: Ciências Exatas e Tecnológicas, Vol. 32, n. 2, p. 165-174, 2012.

[9] ANTONELLI, Jhonatas. Utilização de madeira residual de pinus na adsorção de chumbo e posterior gaseificação e geração de energia elétrica. 2015. 61p. Dissertação (Programa de Pós-Graduação em Engenharia de Energia na Agricultura - Mestrado), Universidade Estadual do Oeste do Paraná: Cascavel, 2015

[10] LEVY, R.; Carbon monoxide pollution and neurodevelopment: a public health concern. Neurotoxicology and Teratology, Vol. 49, p. 31-40, 2015.

[11] SANDILANDS, E.A.; BATEMAN, D.N.; Carbon monoxide. The American Journal of Medicine, Vol. 44, n. 3, p. 151-152, 2016.

[12] TSAI, C. F.; YIP, P. K.; CHEN, S. Y.; LIN, J. C.; YEH, Z. T.; KUNG, L. Y.; WANG, C.Y.; FAN, Y. M.; The impacts of acute carbon monoxide poisoning on the brain: Longitudinal clinical and $99 \mathrm{mTc}$ ethyl cysteinate brain SPECT characterization of patients with persistent and delayed neurological sequelae. Clinical Neurology and Neurosurgery, Vol. 119, p. 21-27, 2014

[13] GORMAN, D.; DREWRY, A.; HUANG, Y. L.; SAMES, C.; The clinical toxicology of carbon monoxide. Toxicology, Vol. 187, n. 1, p. 25-38, 2003.

[14] GUZMAN, J.A.; Poisoning by carbon monoxide. Section of Critical Care, Respiratory. Institute Medical Intensive Care Unit, Vol. 28, n. 4, p. 538-548, 2012

[15] PROCKOP, L. D.; CHICHKOVA, R. I.; Carbon monoxide intoxication: An updated review. Journal of the Neurological Sciences, Vol. 262, n. 1-2, p. 122$130,2007$.

[16] SHEN, M.; FAN, D.; ZANG, Y.; CHEN,Y.; ZHU, K.; CAI, Z.; LIU, Y.; SUN, X.; LIU, J.; GONG, J.; Neuroprotective effects of methane rich saline on experimental acute carbon monoxide toxicity. Journal of the neurological sciences, Vol. 369, p. 361-367, 2016.

[17] KURODA, H.; FUJIHARA, K.; KUSHIMOTO, S.; AOKI, M.; Novel clinical grading of delayed neurologic after carbon monoxide poisoning and factors associated with outcome. Neurotoxicology, Vol. 48, p. 35-43, 2015.

[18] CARDIGA, R; PROENÇA, M.; CARVALHO, C.; COSTA, L.; BOTELLA, A.; MARQUES, F.; PAULINO, C.; CARVALHO, A.; FONSECA, C.; What do we know about carbon monoxide poisoning and cardiac compromise? Treatment and prognosis. Portuguese Journal of Cardiology, Vol. 34, p. 557, 2015

[19] CHANG, Y. C.; LEE, H.Y.; HUANG, J. L.; CHIU, C. H.; CHEN, C. L.; WU, C. T.; Risk factors and outcome analysis in children with carbon monoxide poisoning. Pediatrics and Neonatology, Vol. 58, n. 2, p. 1-7, 2016

[20] SWEENEY, L.M.; SOMMERVILLE, D.R.; GOODWIN, M.R.; JAMES, R.A.; CHANNEL, S.R.; Acute toxicity when concentration varies with time: a case study with carbon monoxide inhalation by rats. Regulatory Toxicology and Pharmacology, Vol. 80, p. 102-105, 2016. 


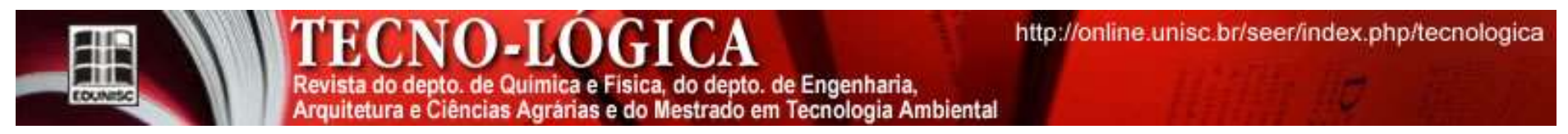

[21] TRENTINI, J. F.; O’NEILL, J.T.; POLUCH, S.; JULIANO, S.L.; Prenatal carbon monoxide impairs migration of interneurons into the cerebral cortex. Neurotoxicology, Vol. 53, p. 31-44, 2015.

[22] BLEECKER, M.L.; Chapter 12- Carbon monoxide intoxication. Handbook of Clinical Neurology, vol. 131, p. 191-203, 2015.

[23] SYKES, O.; WALKER, E.; The neurotoxicology of carbon monoxide Historical perspective and review. Journal of Cortex, Vol. 74, p. 440-448, 2016.

[24] JAMES, P. J. J.; Carbon Monoxide Exposure: Clinical Findings - Sequelae in Survivors. Encyclopedia of Forensic and Legal Medicine (Second Edition), London, p. 461-466, 2016.

[25] SADEGHINIA, M.; REZAEI, M.; AMINI, E.; Preparation of $\alpha-\mathrm{MnO}_{2}$ nanowires and its application in low temperature $\mathrm{CO}$ oxidation. Journal Chemical Engineering, Vol. 30, n. 11, p. 2012-2016, 2013.

[26] PARK, A. J. H.; KANG, D. C. A.; SHIN, C. H.; CO oxidation over $\mathrm{MnO}_{2}$ catalysts prepared by a simple redox method: Influence of the Mn (II) precursors. Journal of Industrial and Engineering Chemistry, Vol. 25, p. 250-257, 2015.

[27] CARRILLO, I.; RAMÍREZ, J.M.; MAGAÑA, L. F.; Adsorption of carbon monoxide, carbon dioxide and methane on hexagonal boron nitride with high titanium coverage. Surface Science, Vol. 637-638, p. 48-52, 2015.

[28] RAMESH, K.; CHEN, L.; CHEN, F.; LIU, Y.; WANG, Z.; HAN, Y.; Reinvestigating the $\mathrm{CO}$ oxidation mechanism over unsupported $\mathrm{MnO}, \mathrm{Mn}_{2} \mathrm{O}_{3}$ and $\mathrm{MnO}_{2}$ catalysts. Catalysis Today, Vol. 131, n. 1-4, p. 477-482, 2008.

[29] GAO, J.; JIA, C.; ZHANG, L.; WANG, H.; YANG, Y.; HUNG, S. F.; HSU, Y. Y.; LIU, B.; Tuning chemical bonding of $\mathrm{MnO}_{2}$ through transition-metal doping for enhanced CO oxidation. Journal of Catalysis, Vol. 341, p. 82-90, 2016.

[30] HU, X.; ZHU, S.; HUANG, H.; ZHANG, J.; XU, Y.; Controllable synthesis and characterization of $\alpha-\mathrm{MnO}_{2}$ nanowires. Journal of Cristal Growth, Vol. 434, p. 7-12, 2016.

[31] WANG, B.; QJU, J.; FENG, H.; WANG, N.; SAKAI, E.; KOMIYAMA, T.; Preparation of $\mathrm{MnO}_{2} /$ carbon nanowires composites for supercapacitors. Electrochimica Acta, Vol. 212, p. 710-721, 2016.

[32] DASH, S.; PATEL, S.; MISHRA, B. K.; Oxidation by permanganate: synthetic and mechanistic aspects. Tetrahedron, Vol. 65, n. 859, p. 707-739, 2009.

[33] RUSEVOVA, K.; KOPINKE, F. D.; GEORGI, A.; Stabilization of potassium permanganate particles with manganese dioxide. Chemosphere, Vol. 86, n. 8, p. 783-788, 2012.

[34] SIGMA. Permanganato de potássio. Disponível em: http://www.sigmaaldrich.com/catalog/product/sigald/223468?lang=pt\&region=B R. Acesso em 10/08/2016.

[35] SIMEPAR, Sistema Meteorológico do Paraná. Condições Atuais: Toledo, pressão atmosférica média, Curitiba. Disponível em http://simepar.br/. Acesso em $20 / 10 / 2016$

[36] YOE, J. H., JONES, A. L. Colorimetric determination of iron with disodium1,2-dihydroxy-3,5-disulfonate. Industrial and Engineering Chemistry Analytical Edition, Vol. 16, n. 2, p. 111-115, 1944.

[37] BALL, D.W. Físico-química. Vol. 2; Pioneira Thomson Learning; São Paulo, 2006

[38] BACCAN, N.; ANDRADE, J. C.; GODINHO, O.E.S.; BARONE, J.S.; Química analítica quantitativa elementar. 3. ed. São Paulo: Edgar Blucher Ltda, 2001.

[39] SKOOG, D. A.; WEST, D. M.; HOLLER, F. J.; CROUCH, S. R.; Fundamentos de Química Analítica; Pioneira Thomson Learning; São Paulo 2006. 999p.
[40] NIST. National Institute of Standards and Technology. Carbon Monoxide. Disponível http://webbook.nist.gov/cgi/cbook.cgi?Formula=CO\&NoIon=on\&Units=SI.

Acesso em 28/03/2017.

[41] LIFENG, C.; DI, Z.; YANG, Y.; YUXIN, W.; XIAODONG, Z.; Synthesis of highly efficient $\alpha-\mathrm{Fe}_{2} \mathrm{O}_{3}$ catalysts for CO oxidation derived from MIL-100(Fe). Journal of Solid State Chemistry, Vol. 247, p.168-172, 2017.

[42] DU, Y.; WANG, X.; WANG, J.; WU, J.; QI, C.; Fabrication of ThreeDimensional Ordered Mesoporous Manganese Oxides Used as High-Efficient Catalysts for Removal of Toluene and Carbon Monoxide. Journal of Nanoelectronics and Optoelectronics, Vol. 12, n. 5, p. 518-525, 2017.

[43] SHIN-ICHI, Y.; ZYUN, S.; MASAFUMI, A.; TSUTOMU, I.; Removal of $\mathrm{CO}$ from CO-contaminated hydrogen gas by carbon supported rhodium porphyrins using water-soluble electron acceptors. Journal of Power Sources, Vol. 329, p. 88-93, 2016. 